\title{
Wave Interaction With Tessellated Metamaterials
}

\author{
Kimberley W. Eccleston \\ Dept of Electrical and Computer Engineering \\ University of Canterbury \\ Christchurch, New Zealand \\ E-mail: kim.eccleston@canterbury.ac.nz
}

\begin{abstract}
This paper considers the interaction of waves with a region comprised of square tiles that display either left-handed or right-handed propagation behaviour arranged in a checkerboard tessellation. This structure exhibits infinite wavelength behavior in two dimensions. Using this structure, interesting wave phenomena can be obtained such as: excitation of uniform plane waves from a point source, redirection of uniform plane waves in one direction, and shielding of occlusions from plane waves with normal incidence. During simulation, the media was modeled as 4-port tiles and each 4-port tile was modeled by either a low-pass or high-pass LC network. The investigations were performed on structures comprising up to 32,000 tiles.
\end{abstract}

Keywords- Metamaterials, tessellated metamaterials, lefthanded materials, negative refractive index, planar circuits, guided waves, uniform plane waves, cylindrical waves.

\section{INTRODUCTION}

The prospect of a planar lens [1] is a well known application of meta-materials and this has driven the microwave community to pursue investigations of metamaterial properties at microwave frequencies. Of particular interest to the microwave community are planar guided-wave structures that are amenable to microstripline circuits [2] - [4]. Initial investigations of two-dimensional (2D) structures [3][4] demonstrated focusing using a slab of left-handed material. More recently, a planar parabolic left-handed refractor [5][6], and a tessellated metamaterial structure [7] have been proposed. The tessellated metamaterial structure exhibited the infinite wavelength phenomenon [8] in 2D which can be used in a square-shaped $\mathrm{N}$-way power divider [9].

The planar lens [1] and parabolic metamaterial refractor [5][6] comprise distinct left-handed and right-handed regions. The tessellated metamaterial [7], on the other hand, use alternating left-handed and righted handed square tiles arranged in a checker-board pattern. The left-handed and right-handed tiles are impedance matched and have equal but opposite phase shift to achieve zero net phase shift between two adjacent tiles [7][9]. Under this condition, the waves exhibit zero phase shift in both directions of the planar structure. In this work we consider other interesting wave phenomena exhibited by the tessellated metamaterial structure when placed in a right-handed medium.

\section{TESSElated METAMATERIAL}

Fig. 1 shows a schematic of the tessellated metamaterial (TMM) structure. In this case, the size of the structure is 7 by 7 but larger or smaller structures are possible. The important feature is the checker-board arrangement of tiles, and at the operating frequency, the insertion phase between any two ports of a tile is $-\varphi$ in the case of the right-handed tiles, and $\varphi$ in the case of the left-handed tiles.

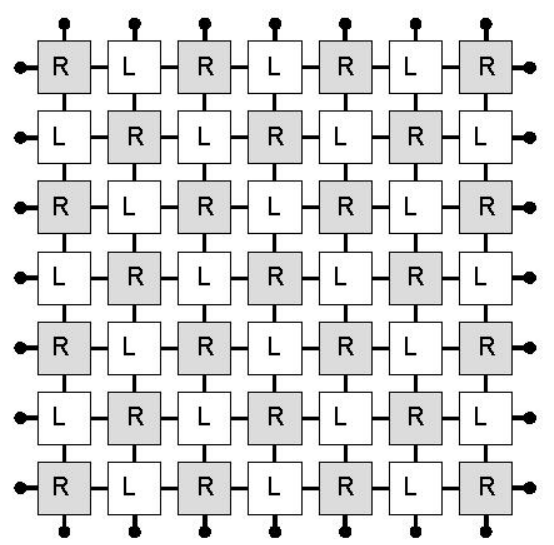

Fig 1. Schematic of the tessellated metamaterial (TMM) structure comprising 4-port square-shaped left-handed (L) and right-handed (R) tiles.

Fig. 2 shows schematics of possible physical realizations of the right-handed and left-handed tiles. The left-handed tile realization is similar to that used by others [3]. If $C=4.37 \mathrm{pF}$ and $L=43.7 \mathrm{nH}, Z_{o}=100 \Omega$ for both tiles, $d_{\mathrm{L}}=4 \mathrm{~mm}$ and $d_{\mathrm{R}}=$ $4.7 \mathrm{~mm}$, then the insertion phase between two ports at $1 \mathrm{GHz}$ of the left-handed and right-handed tile is $11.3^{\circ}$ and $-11.3^{\circ}$ respectively, and the Bloch impedance of both tiles is $100 \Omega$ [7]. Both tiles types can be fabricated so they have the same physical size to enable the tessellation to be physically realised. (a)

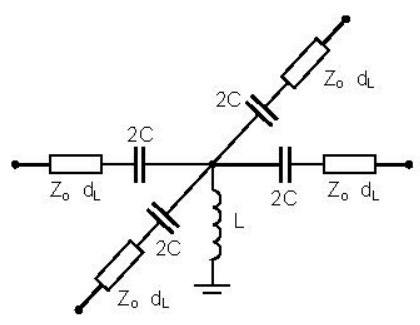

(b)

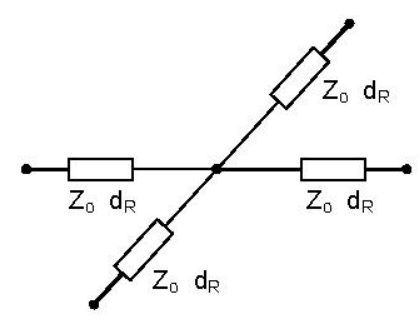

Fig 2. Schematics of physical realizations of: (a) left-handed, and (b) righthanded tiles. 


\section{OVERVIEW OF ANAYSIS METHOD AND VALIDATION}

To simulate and visualize spatial behaviour of waves on large planar structures, Matlab code was developed. The simulation domain was discretised into 4-port square tiles - in essence a tessellation but not to be confused with the TMM. To reduce the computational effort, by reducing nodes, each tile in the planar structure is represented by either a 4-port lowpass or high-pass LC network as shown in Fig. 3. The boundary of the simulation domain is terminated in matched resistances. A graphical interface is used to specify the properties of each tile. The software performs a nodal analysis at a specified frequency and presents the voltage at the centre of each tile in the spatial domain. The number of nodes solved is equal to the number of tiles. To readily identify wave fronts, the cosine of phase as a function of position is presented.

If the LC high-pass tile (Fig. 3(a)) has $\mathrm{C}=8.036 \mathrm{pF}, \mathrm{L}=$ $80.36 \mathrm{nH}$, and the LC low-pass tile (Fig. 3(b)) has $\mathrm{C}=0.318$ $\mathrm{pF}$ and $\mathrm{L}=3.18 \mathrm{nH}$, then at $1 \mathrm{GHz}$, these respectively model the left-handed and right-handed physical tile realizations discussed in section II. The LC low-pass tiles were also used to model large right-handed regions.

(a)

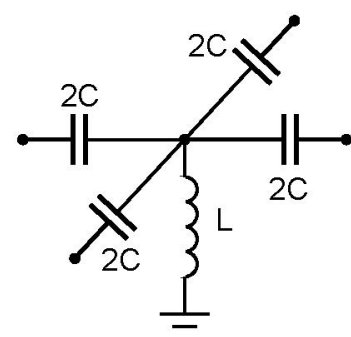

(b)

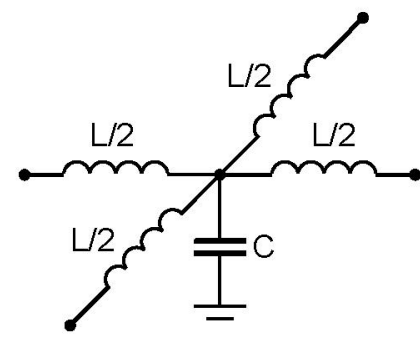

Fig 3. Four-port tiles used during simulation: (a) LC high-pass, and (b) LC low-pass.

Before investigating the behaviour of large planar structures, the computational results of the software were checked against that of a commercial microwave circuit simulator for small structures ( 5 by 5 tiles). Then the validity of the software was validated by simulating the response of large homogenous right-handed regions since the results can be compared with known expected behaviour. A region 200 tiles by 160 tiles with $100 \Omega$ boundary termination resistors was considered. At $1 \mathrm{GHz}$ with the above mentioned LC low-pass tiles, the size of this region is $6.27 \lambda$ by $5.02 \lambda$. Fig. 4 shows the phase response when excited by a point source located at tile position $x=40, y=80$, and is consistent with a cylindrical wave. Well away from the source, standing waves are evident and this is due to residual, albeit very small, mismatch of the tiles at $1 \mathrm{GHz}$ to $100 \Omega$. Fig 5 shows the phase response of this region when excited by a line of point sources (spaced 10 tiles) in a vertical column at $x=40$. The magnitude response (not shown) was essentially flat over the entire domain except near the periphery due to the terminating resistors. Hence, the simulation result is consistent with a uniform plane wave.

Fig. 6 shows the simulated response at $1 \mathrm{GHz}$ of a 19 by 19 tile $(0.6 \lambda$ by $0.6 \lambda)$ TMM of the type shown in Fig. 1 . In this case, phase rather than its cosine has been presented. Since the magnitude and phase variation across this region is only 0.01
$\mathrm{dB}$ and $2.5^{\circ}$ respectively, the tessellated metamaterial displays infinite wavelength phenomena in 2D [7]. Importantly, the results shown in Fig. 6 are consistent with that obtained from a commercial circuit simulator [7] with differences being due to the differing nature of the point source.

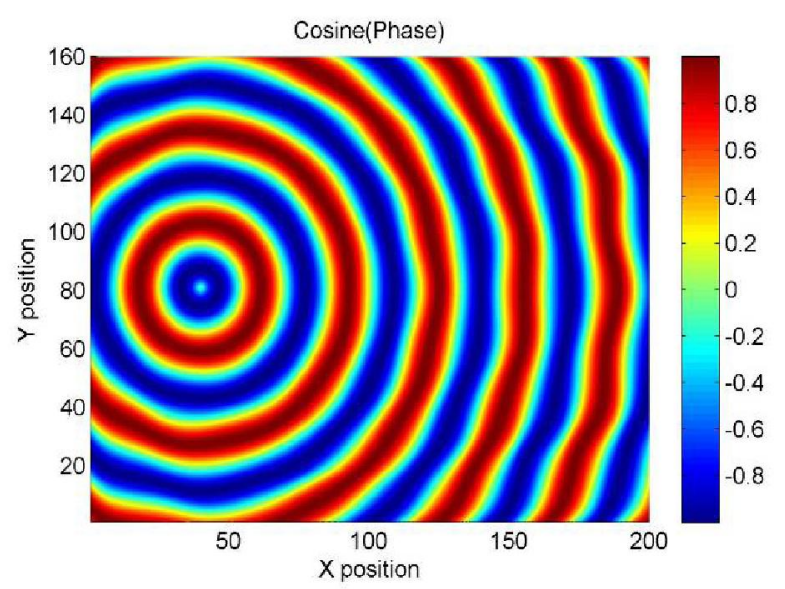

Fig 4. Simulation validation results for a right-handed region excited by a point source at $x=40, y=80$.

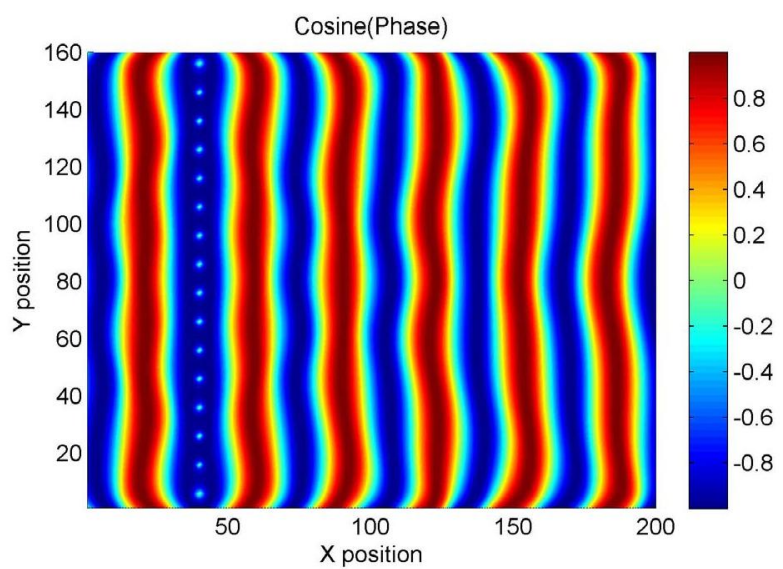

Fig 5. Simulation validation results for a right-handed region excited by a line of 16 point sources along $x=40$.

\section{WAVE INTERACTION WITH A TMM SLAB}

A slab of TMM of the type shown in Fig. 1, 19 tiles wide by 160 tile high, was placed in the right-handed media. The total domain size was 200 tiles by 160 tiles.

In the first example, the slab was placed on one side of the simulation domain. A point source was located in the centre of the TMM slab. Fig. 7 shows the phase response at $1 \mathrm{GHz}$. It can be seen that there is negligible phase shift across the TMM slab and a plane-wave emanates from the side of the slab into the right-handed media. The magnitude response was flat over the entire domain except near the periphery.

It should be mentioned that the phase at the periphery of the TMM slab is not constant but alternates between $\pm 5.6^{\circ}$ about a fixed value from tile to tile [7]. This small alternating phase can be compensated in practice [9] but requires anisotropic tiles and was not considered here due to current software restrictions. 

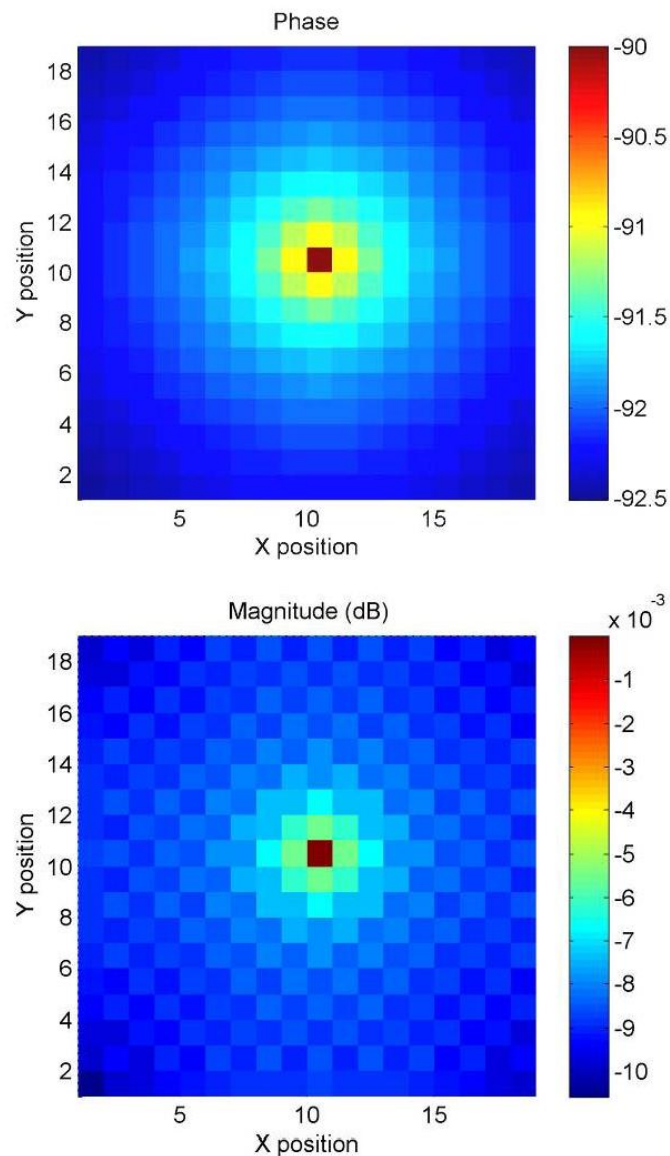

Fig 6. Simulation results for a $19 \times 19$ TMM with point source excitation at the centre.

In the second example, the TMM slab was moved to $x=$ 100 and means that there is a right-handed region on both sides of the slab. A point source was located within one of the righthanded regions at $x=40, y=80$. Fig. 8 shows the phase response. As expected, a cylindrical wave emanates from the point source. The TMM converts this to a plane wave which emanates from the other side of the TMM slab. The magnitude response is flat on other side of the TMM slab. There is reflection from the TMM slab, as evident by the interference pattern on the source side of the slab, and this means that the magnitude of the plane wave transmitted is about $15 \mathrm{~dB}$ lower than the incident wave.

Both example 1 and 2 show that a TMM can transform a point source into a line source, resulting in a uniform plane wave. Moreover the TMM slab has become a line source. Other simulations have shown that this transformation is independent of the distance of the point source from the TMM slab. This is in contrast to a parabolic refractor [5], or a parabolic reflector, where the point source must be located at a focal point.

The third example, a right-handed region is excited by a line of point sources (spaced 10 tiles) in a vertical column at $x$ $=40$. The phase response is shown in Fig. 9. Both the phase and magnitude responses are indicative of a uniform plane wave that passes through the TMM slab with minimal attenuation or reflection.

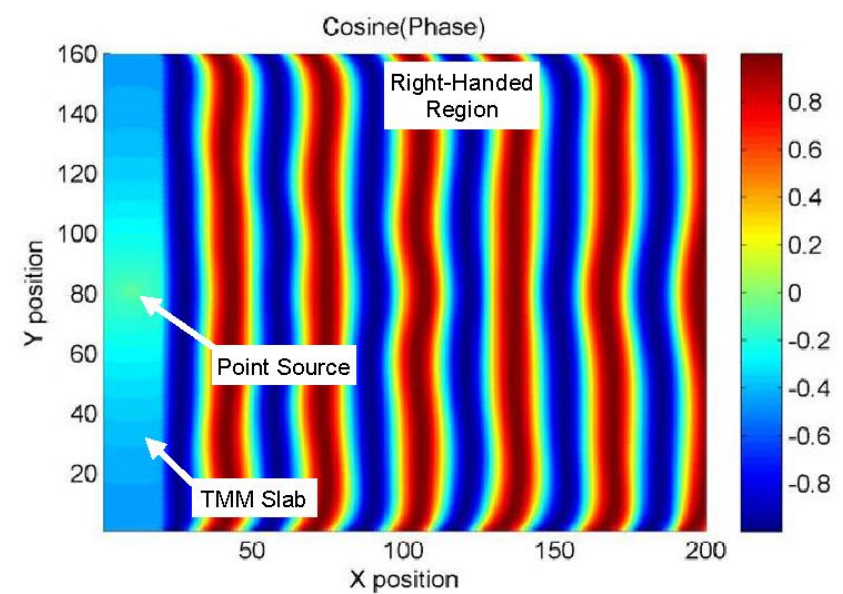

Fig 7. Simulation results for a TMM slab excited by a point source at the centre of the TMM slab.

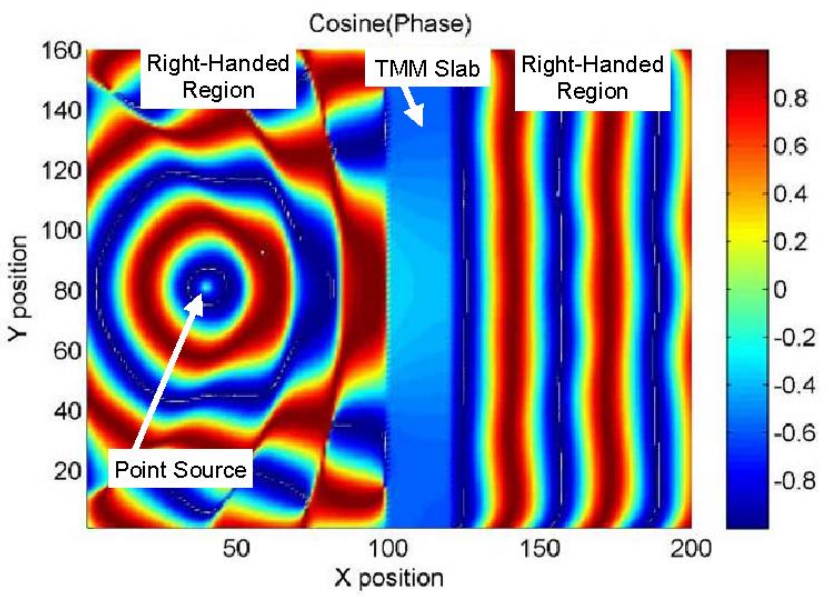

Fig 8. Simulation results for a TMM slab with point source excitation in a right-handed region.

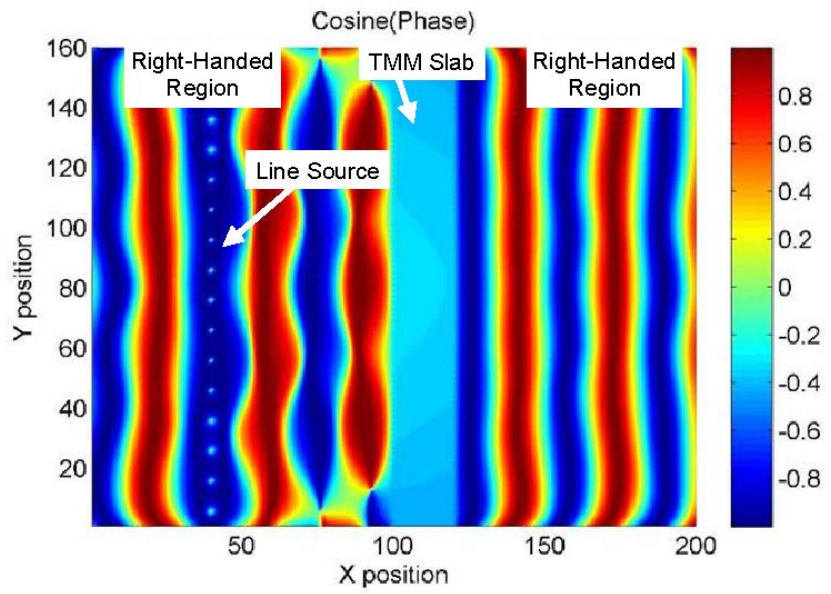

Fig 9. Simulation results for a TMM slab with vertical line source excitation in a right-handed region.

Fig. 10 shows the phase response for a diagonal line source. In this case, a uniform plane emanating from the line source is oblique to the TMM slab, but the wave emanating from the other side of the TMM slab is normal to it. Therefore, the TMM slab has the property that it can redirect a 
uniform plane wave. Similar, to example 2 (incident cylindrical wave), reflection from the TMM slab is evident.

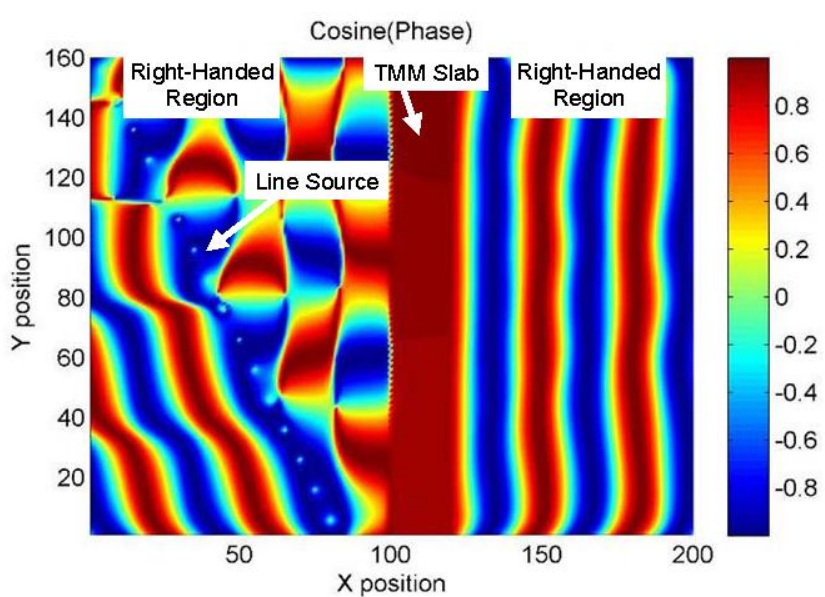

Fig 10. Simulation results for a TMM slab with diagonal line source excitation in a right-handed region.

\section{WAVE INTERACTION WITH AN OCCLUSION EMBEDDED IN A TMM SLAB}

It is well known that metal occlusion will scatter (reflection and diffraction) a wave, and the side facing away from the source will be in shade depending level of diffraction. A conducting occlusion can be modeled by a region of tiles that use the LC low-pass topology with large values of $\mathrm{L}$ and $\mathrm{C}$. Fig. 11 shows the phase response when a conducting slab (occlusion) is embedded in the TMM slab. The magnitude response is essentially flat on the side opposite the line source. So although some reflection has taken place, on the whole, the uniform wave seems to pass through the occlusion undisturbed. Simulations were also repeated in the absence of the TMM to confirm scattering of the occlusion on it is own.

It must be emphasized, however, that this observation is by no means evidence of EM cloaking [11] as only a normal plane uniform wave will pass through undisturbed. Nevertheless, the result depicted in Fig. 11 and the corresponding magnitude response is at least very interesting.

\section{CONCLULSION}

In this paper wave interaction with a slab of tessellated metamaterial (TMM) comprised of right-handed and lefthanded square tiles arranged in a checkerboard pattern, has been investigated. When excited, by any wave, the TMM behaves as a line source and it can be used to transform a cylindrical wave into a uniform plane wave or redirect an oblique plane wave into that normal to the slab. A normal incident plane wave can pass undisturbed through a TMM slab even with an occlusion embedded in it. It would appear that the voltage across the TMM is an "average" of the incident wave at the TMM boundary. So for a normal uniform plane wave, the voltage across the TMM will be equal to the incident wave voltage. Whereas for an oblique uniform plane wave or a cylindrical wave, the TMM voltage will be less than the incident wave voltage and hence reflection and less than 100 percent transmission will occur.

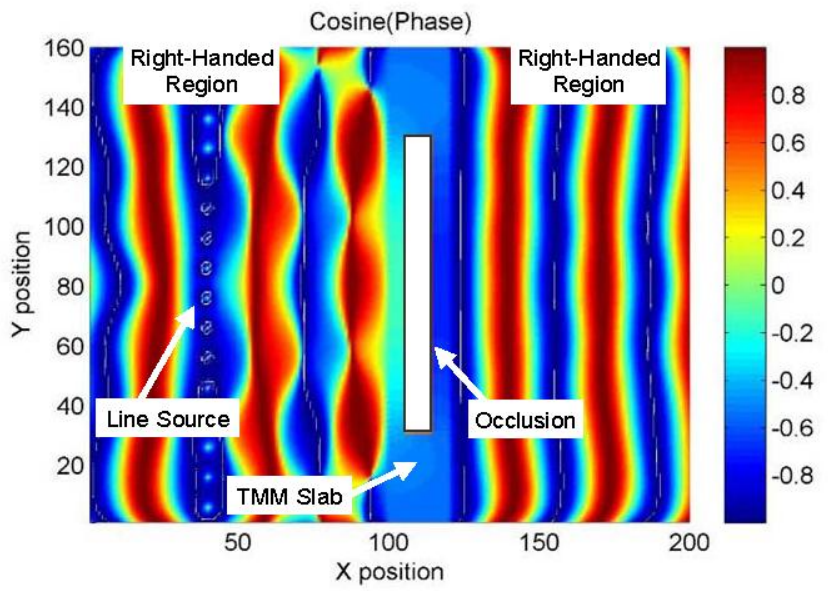

Fig 11. Simulation results for a uniform plane wave normal incident on a conducting occlusion embedded in a TMM slab.

\section{ACKNOWLEDGEMENT}

The author would like to acknowledge the contribution of former University of Canterbury student Mr C. F. Wilson for developing and testing the Matlab code used in this work.

\section{REFERENCES}

[1] J. B. Pendry, "Negative Refraction Makes a Perfect Lens", Physical Review Letters, 85(18), pp 3966 - 3969, 30 Oct. 2000.

[2] C. Caloz and T. Itoh, "Application of the transmission line theory of lefthanded $(\mathrm{LH})$ materials to the realization of a microstrip "LH" line", 2002 IEEE AP-S Int. Symp., pp 412 - 415.

[3] G. V. Eleftheriades, A. K. Iyer and P. C. Kremer, "Planar Negative Refractive Index Media Using Periodically L-C Loaded Transmission Lines", IEEE Trans. on Microwave Theory and Techniques, 50(12), pp $2702-2712$, Dec. 2002

[4] A. Sanada, C. Caloz and T. Itoh, "Planar Distributed Structures With Negative Refractive Index", IEEE Trans. on Microwave Theory and Techniques, 52(4), pp 1252 - 1263, April 2004.

[5] C. Caloz, A. Lai, and T. Itoh, "Wave Interactions in a Left-Handed Mushroom Structure", 2004 IEEE AP-S Int. Symp., pp 1403 - 1406.

[6] A. Lai, W-Y Wu, K. M. K. H. Leong, T. Itoh and C. Caloz, "QuasiOptical Manipulations of Microwaves Using Metamaterial Interfaces", 2005 IEEE AP-S Int. Symp., pp 273 - 276.

[7] K. W. Eccleston, "Investigation of a Tessellated Meta-Material Planar Circuit", 2006 Asia Pacific Microwave Conference, pp 927 - 930.

[8] A. Lai, K. M. K. H. Leong and T. Itoh, "A Novel N-Port Series Divider Using Infinite Wavelength Phenomena", 2005 IEEE MTT-S Digest, pp $1001-1004$.

[9] K. W. Eccleston, "N-way microwave power divider using twodimensional meta-materials", Electronics Letters, 42(15), 20 July 2006, pp $863-864$.

[10] M. A. Antoniades and G. V. Eleftheriades, "Compact Linear Lead/Lag Metamaterial Phase Shifters for Broadband Applications", IEEE Antennas and Wireless Propagation Letters, Vol. 2, pp 103 - 106, 2003.

[11] J. B. Pendry, D. Schurig and D. R. Smith, "Controlling Electromagnetic Fields", Science, Vol. 312, 23 June 2006, pp 1780 - 1782 